\title{
Kinetics of circulating cell-free DNA for biomedical applications: critical appraisal of the literature
}

\author{
Sonia Khier*,1,2 \& Laura Lohan ${ }^{1}$ \\ ${ }^{1}$ Department of Pharmacokinetics, Univ Montpellier, Montpellier, France \\ ${ }^{2}$ IRCM, Inserm, Univ Montpellier, ICM, Montpellier, France \\ * Author for correspondence: sonia.khier@umontpellier.fr
}

Circulating cell-free DNA is considered as one of the major breakthroughs in the field of innovative diagnosis, used as a liquid biopsy. The kinetic parameters of a biomarker are mandatory to assess its usefulness as a diagnostic tool. Obtaining precise mathematical values for the kinetic parameters (e.g., half-life) is then crucial because it could be used for therapeutic monitoring as a prognostic factor. However, little is known about the intrinsic properties of circulating cell-free DNA, more especially, its kinetic properties within the organism. We summarized the basic principles that may affect the kinetics of circulating cellfree DNA within the organism in the light of biological and clinical evidence. We also meta-analyzed the reported data in the literature and the methodologies that have been used to study the kinetic parameters of human circulating cell-free DNA in vivo.

Lay abstract: Circulating cell-free DNA as a biomarker was a major breakthrough in the field of diagnostics. Understanding the kinetic parameters of a biomarker is mandatory to assess its usefulness as a diagnostic tool, especially for therapeutic monitoring. However, at the present time little is known about its kinetic properties within the organism. This review provides an overview of the basic principles that may impact the kinetics of cell-free DNA within the organism and analyzes the reported data thus far.

First draft submitted: 9 November 2017; Accepted for publication: 30 January 2018; Published online: 23 February 2018

Keywords: biomarkers $\bullet$ cell-free DNA $\bullet$ half-life $\bullet$ personalized medicine $\bullet$ pharmacokinetics

Circulating cell-free DNA (cfDNA) is derived from cells in the blood following different mechanisms such as cell death, active secretion, ETosis, phagocytosis, autophagocytosis, etc. [1,2]. Increases in cfDNA levels is described in various pathologic conditions such as cancer, sepsis, autoimmune diseases and in particular, physiological states such as pregnancy or intense physical exercise. As a result, the cfDNA level is studied as a potential biomarker for early diagnosis, diagnosis and prognosis. As a potential biomarker, it is crucial to characterize the intrinsic kinetics properties of cfDNA within the organism, especially when using cfDNA analysis as longitudinal diagnostic tool for monitoring the course of treatment. As an endogenous biological product, cfDNA may follow the physiological distribution (in tissues and physiological fluids) and elimination processes. Kinetic parameters are established when analyzing the evolution of blood, serum or plasma concentrations of a substance over time. Thus, the evolution of the cfDNA concentration over time reflects those processes. Intra- or inter-individual variability due to particular physiological or physiopathological conditions may affect the level of cfDNA. Obtaining precise mathematical values for the kinetic parameters is then crucial because these values could be the reflection of the progression or the regression of a pathology, yet also be a prognostic factor for treatment. While several works were interested in the biodistribution and degradation of genomic DNA throughout the organism, few works have been interested in determining the biodistribution and elimination of cfDNA stricto sensu. 


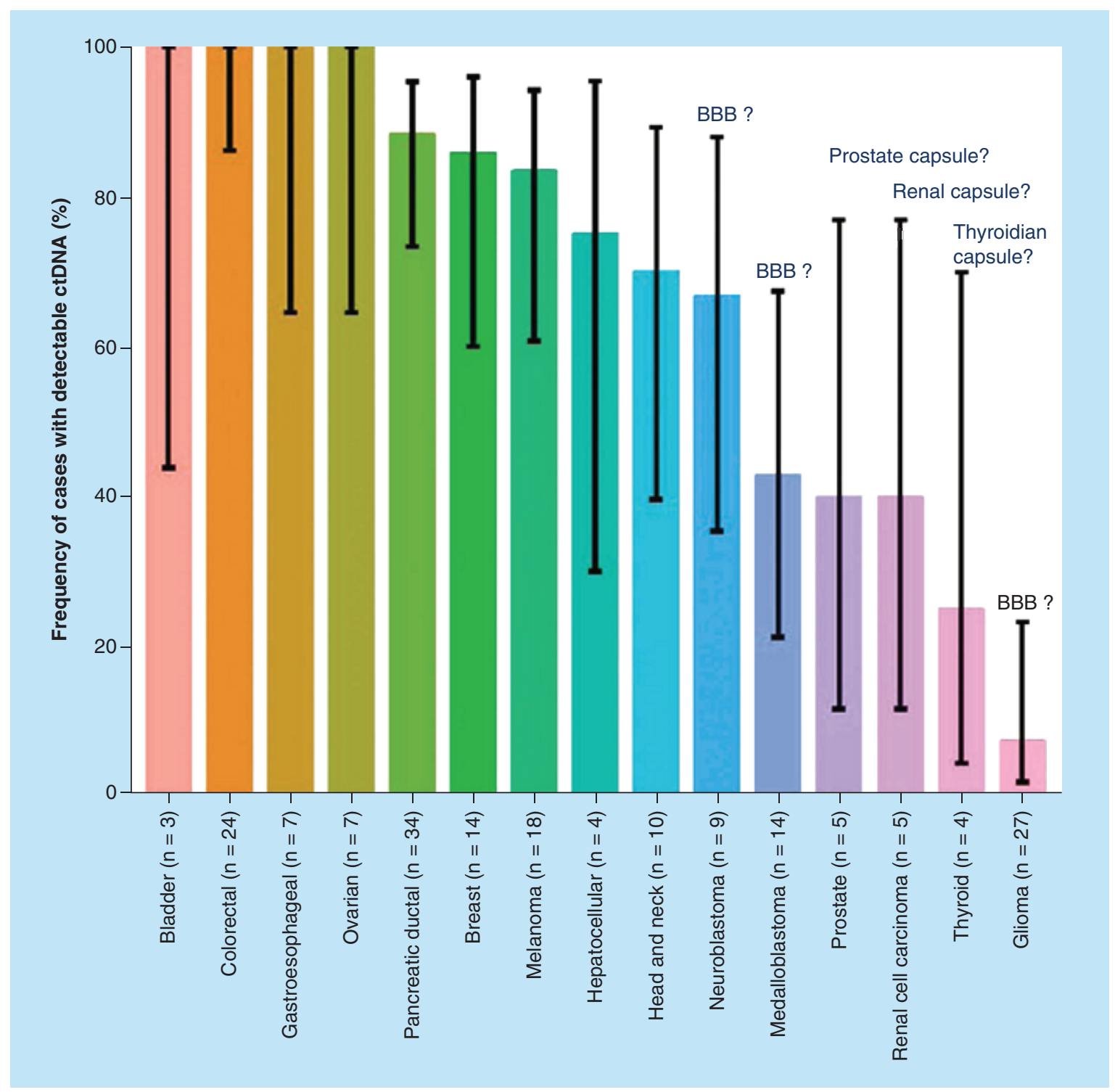

Figure 1. Physiological barriers seem to affect the distribution of tumor-derived cell-free DNA. BBB: Blood-brain barrier; ctDNA: Circulating tumor DNA.

Reproduced with permission from [4] (c) The American Association for the Advancement of Science (2018).

\section{Physiological \& pathological processes responsible for the cfDNA rate} cfDNA release into the blood

cfDNA is released into the blood following cell death mechanisms and/or active secretion. During pregnancy, in addition to their own cfDNA, fetal cfDNA (f-cfDNA) circulates in the blood of pregnant women [3]. In cancer patients, there is a fraction of cfDNA derived from the tumor. It appears that the level of cfDNA in cancer patients depends on the location of tumor. Currently, colon, gastroduodenal tract, breast, pancreas, liver and skin cancers are clearly identified as releasing large amounts of cfDNA within blood while glioma and thyroid tumors release small amounts of cfDNA [4]. We can hypothesize that cfDNA does not cross the blood-brain barrier or the capsule protecting organs such as thyroid, prostate or kidneys (Figure 1) but can easily cross placental barrier. Much like drugs, these physiological barriers may affect the distribution (and the rate) of cfDNA throughout the organism. 


\section{cfDNA distribution throughout the organism}

It is well known that cfDNA is present in human plasma as well as in other biological fluids (urine, feces, cerebrospinal fluid and milk [2]). cfDNA is also present in interstitial spaces [5]. This can be explained by the fact that all cells release cfDNA. However, cfDNA has been shown to be an intercellular messenger and this suggests that it can travel from one site to another within the organism [1].

\section{Evidence based on cfDNA messenger capacity}

M Stroun, P Anker and P Gahan were the first to show the DNA mobility within an organism or between organisms [6-11]. They hypothesized that nucleic acids were active carriers of the genetic information between cells [12]. Various uptake mechanisms can be hypothesized: they can be mediated by extracellular receptors for cfDNA such as TLR9 or HGMB1 [13], with the complexes formed between cfDNA and plasma proteins favoring their internalization using protein receptors [14]. In addition, nucleosomes have been shown to be able to cross the cellular membrane [15], while exosomes can undergo endocytosis [16]. Nevertheless, all those observations are based on in vitro experiments or in vivo experiments using cfDNA derived from in vitro experiments.

\section{Evidence based on identified cfDNA structures}

A significant amount of cfDNA has been shown to be associated with the extracellular surface of blood cells (cell-surface-bound cfDNA [csb-cfDNA]), both erythrocytes and leukocytes due to the presence of nucleic acidbinding proteins $[17,18]$. The cfDNA distribution space could also include the lymphatic system, implying a volume of distribution greater than blood. In addition, cfDNA structures seem heterogeneous (ssDNA or dsDNA fragments from dozen to thousands bp, macromolecular structures such as mono- or oligo-nucleosomes, neutrophil extracellular traps [NETs], nucleolipidoproteic complexes or as microvesicular structures such as microparticles [200-1000 nm] or apoptotic bodies $[1-5 \mu \mathrm{M}][19,20])$. All these cfDNA structures can be present on a cell's surface at the same time. The binding can be reversible, saturable and can also promote internalization of DNA in blood cells [20]. We can hypothesize that free cfDNA or microvesicles could pass through the sinusoidal capillaries to reach other organs such as bone, liver and spleen.

Altogether, these evidences reveal that cfDNA is distributed within the organism subsequent to its release within the blood (Figure 2).

\section{cfDNA elimination process}

cfDNA elimination pathway

Data obtained from animal studies are difficult to extrapolate to humans as the DNA used in these studies is injected, highly purified and comes from different species. However, all these preclinical experiments provide a preliminary insight into the elimination process and show that DNA is rapidly degraded by nucleases present in the blood and rapidly eliminated by the liver and kidneys [21-23]. Lo et al. [24] subsequently performed an in vitro assay to experiment nucleases' role on f-cfDNA clearance from maternal plasma. They concluded that plasma nuclease only plays a partial role in the removal of f-cfDNA in most subjects, and that other organ systems are involved. The experiments carried out by Yu et al. [25] led to similar conclusion derived from in vivo experiments on pregnant women: neither plasma nucleases nor the kidney were the major routes for $\mathrm{f}$-cfDNA elimination. While we cannot confirm the role of DNase activity and renal excretion in the human cfDNA elimination process, we can nevertheless expect them to play a role in the elimination pathway, even if it is minor. As cfDNA can be excreted in urine, recent works have explored detection of cfDNA in urine as a potential 'liquid biopsy' [26-28].

\section{Factors affecting cfDNA elimination}

As already mentioned above, while the eclectic structure of cfDNA present in human blood has an impact on its distribution, it can also have an impact on its elimination. In a pathological context, such as systemic lupus, the presence of a large amount of complex (cfDNA with monoclonal autoantibody) in plasma could have an impact on the half-life (HL) elimination value by decreasing the clearance resulting from a problem of DNases accessibility. Complex cfDNA structures such as nucleosomes, mono- or oligo-nucleosomes, virtosomes, by preventing access to DNases, could in the same way complicate enzymatic activity by nucleases. A lack or diminution of DNase activity could also influence the cfDNA elimination rate. This has been pointed out within a pathological context with systemic lupus $[29,30]$ and cancer. The DNA hydrolyzing activity reveals a low activity in cancer patients and a high blood plasma activity in healthy donors [31]. 


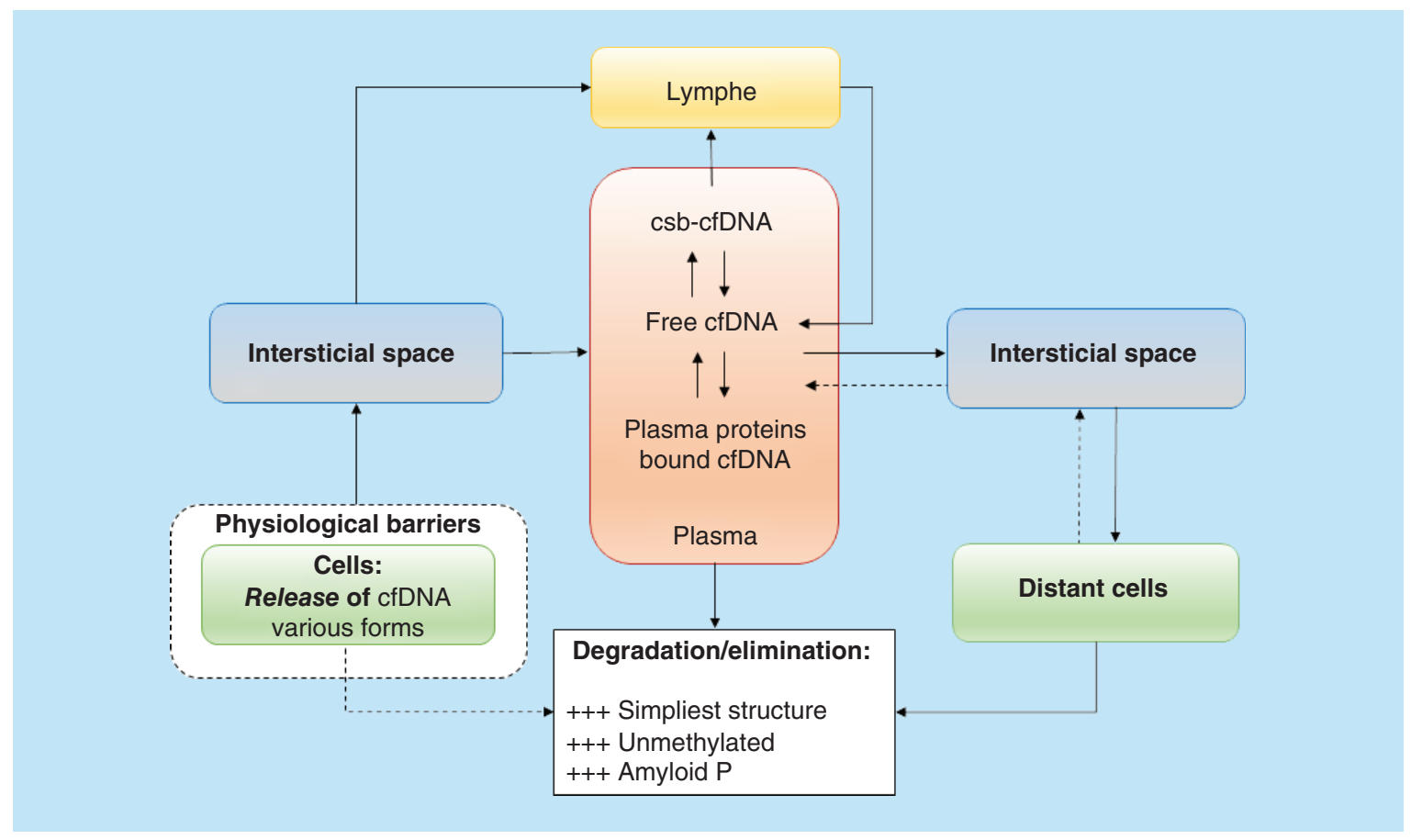

Figure 2. Illustration of cfDNA distribution within the organism based upon identified processes and raised questions.

cfDNA: Cell-free DNA; csb-cfDNA: Cell-surface-bound cfDNA.

At last, the third factor influencing elimination rate is the binding to plasma proteins. The human serum albumin binding was shown to result in long-term cfDNA plasma circulation [32]. The result is an increase in the elimination HL. Skvortsova et al. [33] postulated that an efficient binding of methylated DNA with blood proteins could explain a slower degradation of methylated DNA compared with an unmethylated DNA. On the other hand, studies revealed that serum amyloid P-component might be involved in one of the mechanisms responsible for effective cfDNA clearance inasmuch as it binds cfDNA [34]. It seems that the involvement of plasma proteins in the elimination process depends on the class of plasma proteins interacting with cfDNA.

Different factors (DNA-hydrolyzing enzymes, the structure of cfDNA and protein interactions in blood) can affect the elimination process. Impact of each factor on elimination speed cannot be quantified yet. Hence, determining a global kinetic parameter remains the best way to study the various rates of cfDNA elimination.

\section{Kinetic parameters}

Of the 99 complete texts potentially eligible for meta-analysis using keyword research, only six original articles [2425,35-38] were selected (Figure 3) and analyzed, assessing a kinetic parameter related to stricto sensu human cfDNA (e.g., endogenous human product). We also included the original article found through a routine review of cancer literature that led us to make this review [39]. The main kinetic parameter investigated is the HL. In Table 1 we summarize results and design trials.

\section{Treadmill exercise}

Intense exercise has been reported to increase cfDNA plasma concentrations [40,41]. As inflammatory markers are enhanced following resistance exercise, Fatouros et al. [42] suggest that cfDNA in plasma might be a sensitive marker for exercise-induced inflammation. Thus, physical exercise was used as a model to study the evolution of cfDNA within the organism. Beiter et al. [37] and Breitbach et al. [38] studied a deliberately induced increase of cfDNA in controlled conditions. Samples were collected from healthy subjects (athletes) during a treadmill exercise. Beiter et al. analyzed nuclear cfDNA (88 bp fragment of the chromosomal myostatin gene locus, MSTN) and mitochondrial cfDNA (85 bp fragment of the mitochondrial genome) from venous blood sample by quantitative (q)-PCR. The blood samples were obtained from three individuals during and after the treadmill exercise. A mean 


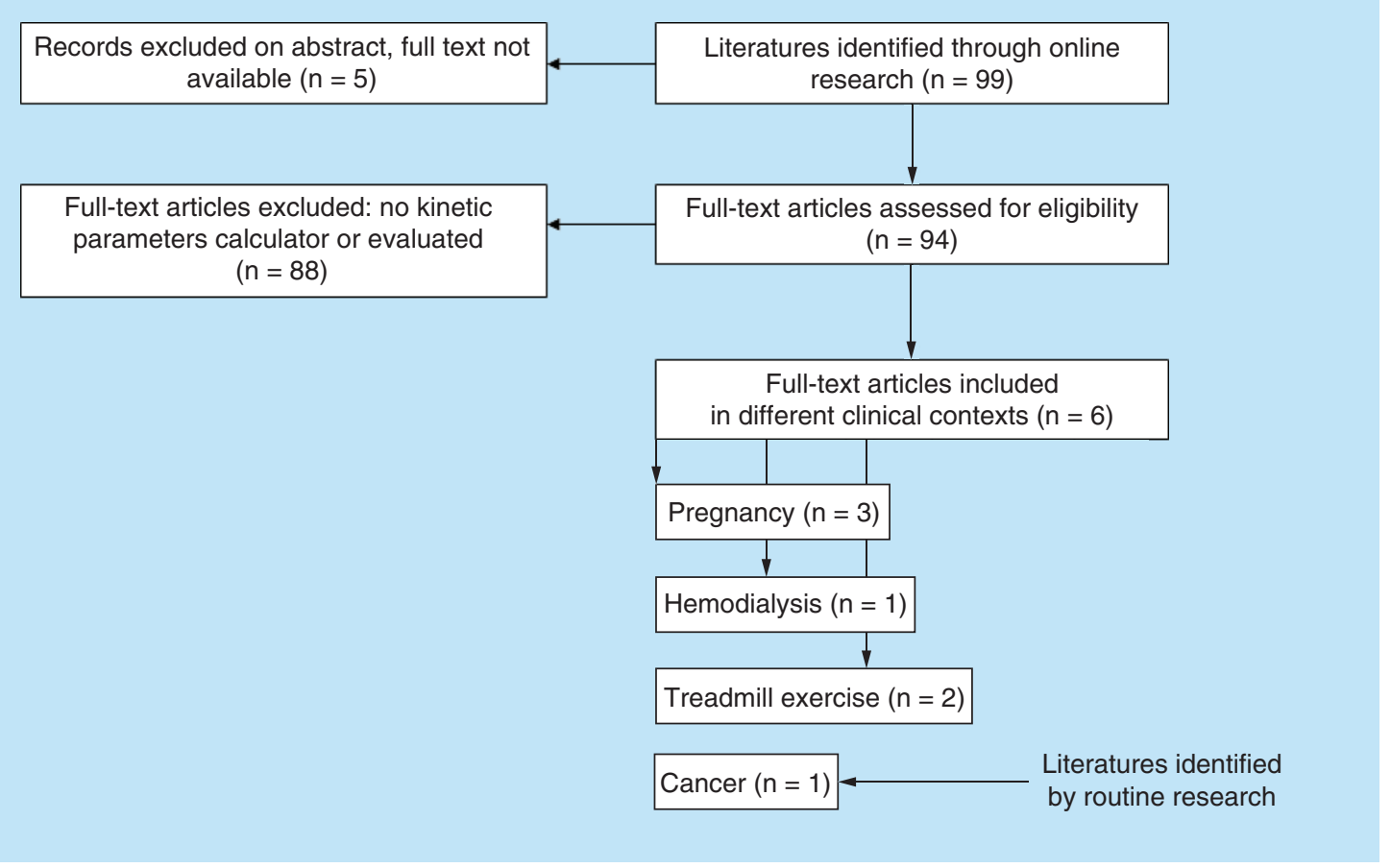

Figure 3. Flow chart of the included studies in the meta-analysis.

HL of 16 min: [range 9-23 min] was obtained. Breitbach et al. applied a new analytic procedure requiring a minimal volume of unpurified plasma for quantification. Capillary blood was collected from the fingertip from 26 athletes (amplification of a 90- and a 222-bp multilocus L1PA2 sequence by q-PCR). During discussion, the authors assumed that a mean HL rate of approximately 15 min could be verified.

\section{Hemodialysis}

DNA is considered to be the antigenic precursor of DNA-anti-DNA immune complexes. The DNA elimination rate would be expected to affect the formation and pathogenicity of these immune complexes. Rumore et al. [35] chose hemodialysis as a model of cfDNA release and proposed determining the elimination rate. During hemodialysis, cfDNA may be released from cells trapped within the dialysis coil. Since that release presumably ceases with the termination of the procedure, it offers an opportunity to estimate the elimination rate of cfDNA in humans with controlled conditions and minimize the interindividual variability of the release (no traumatic release). Blood samples were obtained during the 10 -min posthemodialysis from 15 patients undergoing routine hemodialysis. A mean HL of 4 min was obtained.

\section{Cancer}

Blood concentration of cfDNA proved to be high under several pathological conditions such as cancer. Diehl et al. [39] applied a highly sensitive approach to quantify tumor cfDNA in plasma samples from subjects undergoing multimodality therapy for colorectal cancer. They proposed to use a cfDNA concentration to monitor the tumor dynamics of patients with metastatic colorectal cancer under surgery and chemotherapy. A total of 162 plasma samples from the 18 subjects were collected before and after surgery and a q-PCR on APC, KRAS, PIK3CA or RAS was realized. A HL of 114 min was obtained from one subject among 18.

\section{Pregnancy}

The population within which the kinetic parameter of human cfDNA has been the most studied is that of pregnant women. Since the discovery of f-cfDNA in the maternal blood [3], a number of clinical applications involving the use of this f-cfDNA have been proposed [43]. These include prenatal diagnostic screening and could also serve as a prognostic biomarker for maternal pathologies. Lo et al. [24] were the first to determine a quantitative kinetic 
Table 1. Half-life of circulating cell-free DNA calculated in different contexts.

\begin{tabular}{|c|c|c|c|c|c|c|}
\hline N patients ${ }^{\dagger}$ & $\begin{array}{l}\text { Half-life value - dispersion } \\
\text { parameter }\end{array}$ & $\begin{array}{l}\text { N sample points for } \\
\text { calculation } \ddagger \\
\text { [first point; last point] }\end{array}$ & Calculation method $\S$ & $\begin{array}{l}\text { Total of sample points } \\
\text { obtained per patient } \mathbb{I} \\
\text { between }\left[t_{0} ; t_{f}\right]\end{array}$ & Study & Ref. \\
\hline \multicolumn{7}{|c|}{ Treadmill exercise (q-PCR : MSTN) } \\
\hline 3 & $\begin{array}{l}16 \text { min (mean) - } \\
\text { range: }[9-23]\end{array}$ & $\begin{array}{l}\text { NA } \\
{[N A ; N A]}\end{array}$ & NA & $\begin{array}{l}5 \\
\text { [End of } \\
\left.\text { exercise: } t_{0}+30 \mathrm{~min}\right]\end{array}$ & Beiter et al. & [37] \\
\hline \multicolumn{7}{|c|}{ Hemodialysis context (purified DNA radiolabeled) } \\
\hline 15 & $\begin{array}{l}4 \min (\text { mean })-S D= \\
\pm 1.2 \min \end{array}$ & $\begin{array}{l}2 \\
\text { [Mean absolute } \\
\text { concentration at } t_{0 ;} \\
C_{(t=5 \mathrm{~min})} \text { or } C_{(t=10 \mathrm{~min})]}\end{array}$ & $\begin{array}{l}\text { Least squares } \\
\text { Data expressed as a } \\
\text { fraction of the zero } \\
\text { concentration }\end{array}$ & $\begin{array}{l}2 \text { or } 3 \\
\text { [End of hemodialysis; } \\
t_{0}+10 \mathrm{~min} \text { ] }\end{array}$ & Rumore et al. & [35] \\
\hline \multicolumn{7}{|c|}{ Pregnancy - fetal ccfDNA (q-PCR : SRY) } \\
\hline 8 & $\begin{array}{l}16.3 \text { min (mean) - } \\
\text { range: }[4-30]\end{array}$ & $\begin{array}{l}n=N A \\
{[\text { Peak concentration; NA] }}\end{array}$ & $\begin{array}{l}\text { Mean time taken to } \\
\text { reduce the peak } \\
\text { plasma fetal DNA } \\
\text { concentration by } 50 \%\end{array}$ & $\begin{array}{l}7 \\
\text { [Cesarean; } \\
\left.t_{0}+120 \mathrm{~min}\right]\end{array}$ & Lo et al. 1999 & [24] \\
\hline 7 pre-eclampsie & $\begin{array}{l}114 \text { min (median) - } \\
\text { range: [46-210] } \\
\text { Interquartil: [81-168] }\end{array}$ & $\begin{array}{l}\mathrm{n}=3-7 \\
{\left[\text { Peak concentration }{ }^{\#} ; \text { first }\right.} \\
\text { non-zero trough"\# }{ }^{\#}\end{array}$ & $\begin{array}{l}\text { Log-linear regression } \\
\text { (SigmaStat 2.0) }\end{array}$ & $\begin{array}{l}8 \\
\text { [Cesarean; } \\
\left.t_{0}+360 \mathrm{~min}\right]\end{array}$ & Lau et al. 2002 & [36] \\
\hline 9 control & $\begin{array}{l}28 \text { min (median) - } \\
\text { range: [7-114] } \\
\text { Interquartil: [16-47] }\end{array}$ & & & & & [36] \\
\hline 8 & $\begin{array}{l}0.9 \mathrm{~h}(\text { mean })- \\
\text { range: }[0.6-1.2]\end{array}$ & $\begin{array}{l}n=4-6(n s) \\
\text { [Peak concentration (ns); } \\
120 \mathrm{~min}]\end{array}$ & Log-linear regression & $\begin{array}{l}6-7 \\
{\left[\text { Cesarean; } t_{0}+24 \mathrm{~h}\right]} \\
\text { cases } 1-3 \\
{[\text { Cesarean; }} \\
\left.\mathrm{t}_{0}+120 \mathrm{~min}\right] \text { cases } 4-5 \\
{\left[\text { Cesarean; } \mathrm{t}_{0}+18 \mathrm{~h}\right]} \\
\text { cases } 6-8\end{array}$ & Yu et al. 2013 & [25] \\
\hline 3 (among the 8) & $\begin{array}{l}12.6 \mathrm{~h}(\text { mean })- \\
\text { range: }[4.2-18.1]\end{array}$ & $\begin{array}{l}\text { NS }=2-3(n s) \\
{[6 h ; 18 h] \text { cases } 6-8}\end{array}$ & & & & [25] \\
\hline \multicolumn{7}{|c|}{ Colorectal tumor (q-PCR: TP53). } \\
\hline 1 & $114 \min -\mathrm{NA}$ & $\begin{array}{l}\mathrm{n}=4 \\
{\left[\mathrm{C}_{\text {Day } 1} ; \mathrm{C}_{\text {Day3 }}\right]}\end{array}$ & $\begin{array}{l}\text { Non linear regression } \\
\text { (LM algorithm) }\end{array}$ & $\begin{array}{l}8 \\
\text { [Surgical resection; } \\
t_{0}+\sim 350 \text { days] }\end{array}$ & Diehl et al. 2008 & [39] \\
\hline \multicolumn{7}{|c|}{$\begin{array}{l}\text { For all the studies, plasma was the sample matrix. } \\
\text { †Number of patients included in the analysis. } \\
\text { ¥Number of plasma samples used for calculation of half-life. } \\
\text { § } \text { Method used to evaluate half-life. } \\
\text { I Total number of samples obtained from patient along the study, baseline included. } \\
\text { \# } 5 \text {-45 min post-delivery. } \\
\text { \#\# } 45-360 \text { min post-delivery. } \\
\text { cfDNA: Circulating cell-free DNA; NA: Not available; ns: Not specified in the text, the values were evaluated from concentration-time curves; q-PCR: Quantitative PCR; SD: Standard } \\
\text { deviation; } t_{0} \text { : Beginning of the kinetics, from this time a decline of cfDNA concentration is expected; tf: Time of the last sample obtained during assay. }\end{array}$} \\
\hline
\end{tabular}

parameter of f-cfDNA from maternal plasma. After delivery (cesarean) maternal peripheral blood was collected at different times from eight women (free of any medical disease or any antenatal complications). f-cfDNA was quantified by q-PCR, targeting a sequence of 198 bp on $S R Y$ (only male fetuses were selected). The mean time taken to reduce the peak plasma f-cfDNA concentration by $50 \%$ was 16.3 min (range: 4-30 min) representing mean plasma HL. While the f-cfDNA elimination process from the maternal plasma may appear to be rapid, a number of pathological conditions associated with damage to and dysfunction of the kidneys have been associated with the impaired clearance of f-cfDNA. For this reason, Lau et al. [36] proposed describing the kinetics of f-cfDNA in the plasma of seven pre-eclamptic women versus nine control women in the same condition as Lo et al. (post-cesarean sampling, q-PCR, SRY). As the f-cfDNA concentration in maternal plasma is higher in pre-eclamptic women than in pregnant control women of the same gestational age, they hypothesized that a slower maternal elimination process could explain the different level of $\mathrm{f}$-cfDNA concentration. A median HL of 28 min (range: 7-114 min) was evaluated for the control group and a median HL of $114 \mathrm{~min}$ [range 46-210 min] for the pre-eclamptic group. $\mathrm{Yu}$ et al. [25] proposed identifying the major f-cfDNA elimination route (renal or nucleases elimination) using a precisely quantified f-cfDNA $(S R Y)$. For eight women with uncomplicated singleton pregnancies, the number of 
samples was sufficient to determine a concentration versus time curve. A median HL of $0.9 \mathrm{~h}$ (range: $0.6-1.2 \mathrm{~h}$ ) was evaluated. But for three women among the eight, the sampling protocol was different and late sampling points have been integrated in the concentration versus time curve. For these three women, two phases of decreased concentration were observed, leading to a median HL evaluation for the 'rapid phase' of $0.9 \mathrm{~h}$ (range: $0.6-1.2 \mathrm{~h}$ ) and an HL for the 'slow phase' of $12.6 \mathrm{~h}$ (range: 4.2-18.1 h).

\section{Discussion}

\section{Assessment of kinetic parameters}

A parallel can be drawn between pharmacokinetic (PK) parameters obtained for a drug and the kinetic parameters of a biomarker/endogenous product. This is because these entities can pursue the same physiological biodistribution and elimination stages and because the parameters used to describe the kinetics are the same. The methodologies used to obtain PK parameters are presented as supplementary data.

\section{Physiological cfDNA release}

Beiter et al. [37] studied the time-concentration course of cfDNA in controlled conditions (healthy athletes) during a treadmill exercise. These two cases are particularly interesting because they permit to study cfDNA under basal conditions (no pathologies) and its release with a minimal source of variability. Despite these conditions, the concentrations versus time profile is very different from one subject to another (see multipanel curves in Supplementary Data). It is worthwhile noting that plasma mitochondrial cfDNA concentrations were not affected by the treadmill exercise. For the kinetic protocol, series of blood samples from three participants were collected without any further information concerning the method or study design used for the kinetic analysis. Within the same context (treadmill exercise), Breitbach et al. [38] discuss a rapid increment of cfDNA concentrations and that "in 50\% of the subjects in the present study, the mean HL rate of approximately 15 min could be verified", for a total of 26 subjects included in the study, without any further information concerning the calculation method or study design for the kinetic analysis. It would be interesting to obtain more accurate results, and more information about design protocol and methodology.

Rumore et al. [35] chose the cfDNA released into the plasma during the hemodialysis course as a model. A question was raised concerning the methodology. Blood samples were collected from 15 patients, with 11 blood samples taken at $5 \mathrm{~min}, 13$ blood samples at $10 \mathrm{~min}$ and we assumed 15 blood samples at $\mathrm{t}_{0}$. The slope was calculated with the mean absolute concentration at $t_{0}$ (from the 15 patients) and $t_{5 \text { min }}$ or $t_{10 \text { min }}$ (see multipanel curves in Supplementary Data). The slope could be evaluated with just two values, but this does not match the practices generally used in PK analyses which require a minimum of three values to calculate a slope. Other biases are explained by the author, such as the selection of patients included in the study being dependent on the concentration at $\mathrm{t}_{0}$. The choice of hemodialysis is interesting because it permits elimination to be studied without provoking a peak concentration caused by a traumatic process (pathology, surgery or exercise). However, knowing that the kidney is involved in the elimination of cfDNA (even as a minor route), this implies that the physiopathological status of patients (renal failure) has an impact on the elimination rate and the evaluated HL. In the PK field, patients with disorders concerning elimination pathways are excluded from early studies to study kinetics in a standardized situation.

\section{Interindividual variability}

Pathological states can influence the cfDNA concentration. Csb-cfDNA concentrations and content are both altered in cancer patients. The cell-free to csb-cfDNA ratio in blood depends on the condition of the donor. While the majority of cfDNA from healthy donors is linked to cells, cancer patients possess an altered distribution of cfDNA that depends on type and location of the tumor [20]. As previously mentioned, plasma DNase reveals a lower hydrolyzing activity in cancer patients than in healthy donors [31]. In addition, the protein binding of cfDNA to plasma proteins greatly influences its distribution as protein concentration can be affected by the physiopathology state of donors (hypo-hyper albuminaemia, systemic lupus erythematosus, etc.). These results show different sources of variability of concentration cfDNA.

The results of Diehl et al. [39] highlight the problem of variability in a particular context. They worked on the detection of a cfDNA-specific gene mutation that could be used as a useful biomarker in cancer detection, monitoring and prognostics. The aim of the study was not to determine kinetic parameters and, consequently, the design was not optimized for that purpose. The HL determination was set for one patient, having a smooth 


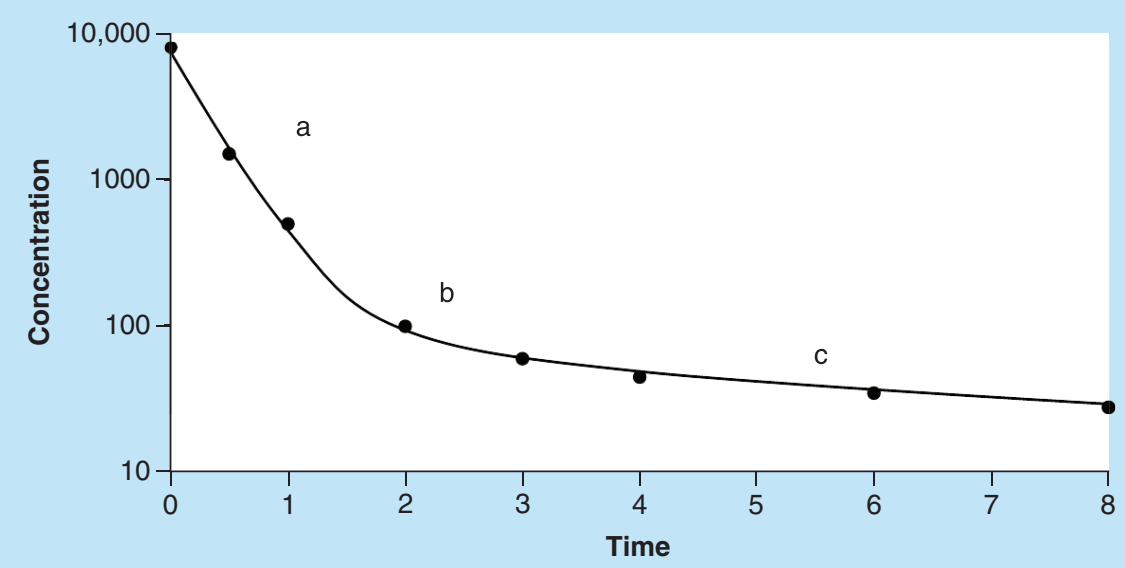

Figure 4. Concentration versus time curve with a shape characteristic of two rate of concentration decrease (two-compartment model). (a) Decreased concentration from the blood due to distribution and elimination process; (b) Inflexion point or pseudo-equilibrium state; (c) Decreased concentration from the blood due to elimination only (log-linear terminal phase); slope permits to calculate half-life of elimination $\left(t_{\lambda z}\right)$.

concentration curve shape. Because the interindividual variability was too high, calculating an HL for the 18 patients included in the study proved difficult (see multipanel curves in Supplementary Data). The causes of variabilities in the curves shape mainly result from the various therapy sources (chemotherapy, surgery, etc.), level of tumor resection and the condition of the patients.

It is now well known that for a same drug administered at the same dosage, the concentration curve could be different from one patient to another and for a same patient having physiopathological variations. The population pharmacokinetics approach (POPPK) is commonly used to analyze the PK parameters of drugs and also to identify and quantify the origins of interindividual variabilities [44-46]. The POPPK approach has been used for the kinetic analysis of several serum tumor biomarkers [47] and could be an interesting approach to treat the variability issue for an endogenous component [48].

\section{Different kinetic stages for cfDNA}

Within the context of pregnancy, Yu et al. [25] monitored cfDNA plasma concentrations from eight subjects over a period of $18 \mathrm{~h}$ and more. A slope was determined by log-linear regression (we assume from the postdelivery

concentration peak to the last sample concentration). The slope is expressed in HL terms by the $H L=\frac{\ln (2)}{\text { slope }}$ relationship, working with the hypothesis of monophasic (monoexponentiel) decrease. But for three patients with a rich data sampling from 2 to $18 \mathrm{~h}$ postdelivery, the shape of the concentration-time curve was modified. The log-representation of the concentration versus time was biphasic for these three patients whereas it was monophasic for the others (see multipanel curves in Supplementary Data). The biphasic curve describes two concentration rate decreases meaning different kinetic stages could be described in an independent manner.

To explain the shape of the curve, the authors hypothesized that different mechanisms could be involved in the elimination process: nucleases for rapid phase and maternal immunity and/or reticuloendothelial systems for the slow phase. However, no substantial change was apparent in the short to long f-cfDNA fragments ratio after delivery. The authors have considered two slopes (the first rapid and the second slow), both representing the elimination phase and expressing them directly in HL using the $H L=\frac{\ln (2)}{\text { slope }}$ relationship. They obtained two kinetic parameters to describe the same elimination process (clearance).

In classical PK analysis, this biphasic shape could be assimilated to a two-compartment model (Figure 4). After the delivery-associated traumatic procedure, the first part of the curve (high slope) could describe the kinetics of the disappearance of the substance from the blood, with the disappearance due to both the distribution and elimination processes. The terminal phase (lower slope) describes the kinetics of disappearance resulting from the 


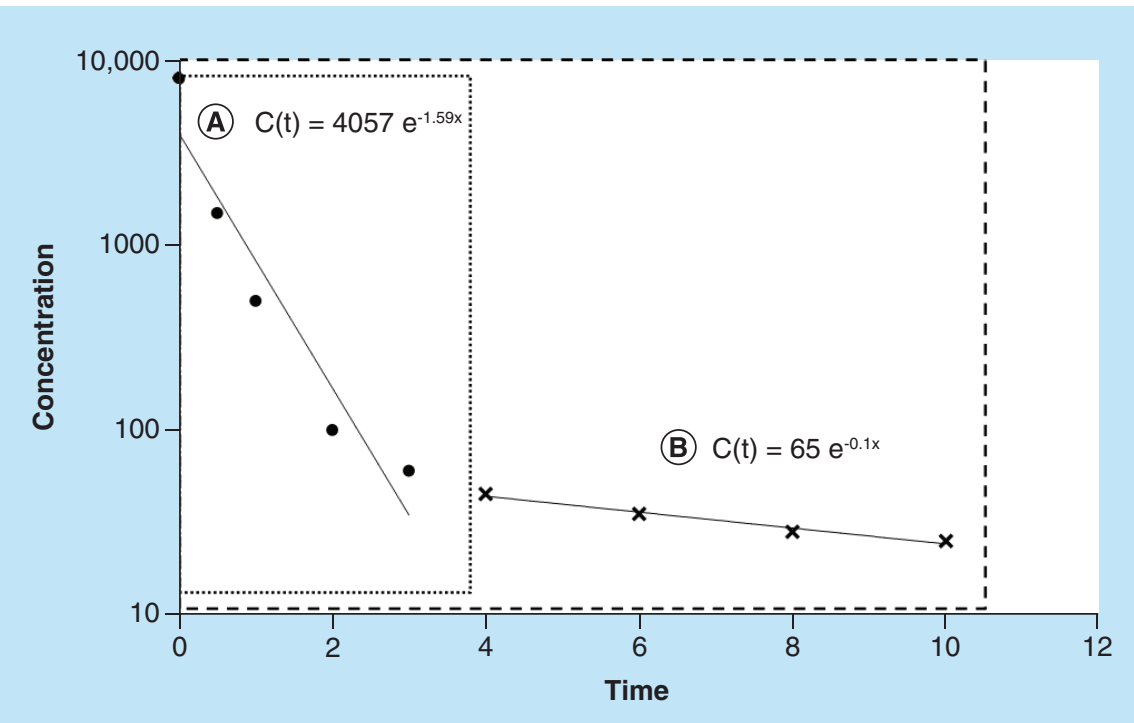

Figure 5. Importance of the window blood sampling. (A) Window blood sampling with $\mathrm{t}_{\mathrm{f}}=3 \mathrm{~h}$ and (B) window blood sampling with $t_{f}=10 \mathrm{~h}$ for the same entity. In the first case (A), the window is too short and the elimination phase is not described alone. The slope $(1.59 \mathrm{~h}-1)$ could be considered as the rate of elimination whereas it is the consequence of distribution and elimination process. If an optimal design is obtained (including later points ' $\times$ ', case [B]), the real elimination rate is close to $0.1 \mathrm{~h}-1$.

$t_{f}$ : Time of the last sample obtained during assay.

elimination process alone and allows the calculation of the elimination HL ( $\left.t_{\lambda z}\right)$, also known as terminal HL or biological HL. The inflexion point reflects the pseudo-equilibrium stage, meaning a homogenate distribution is obtained throughout the organism. Following this reasoning, the biological HL ( $\left.H L=\frac{\ln (2)}{\text { Terminal slope }}\right)$ obtained by $\mathrm{Yu}$ et al. [25] is close to $12.6 \mathrm{~h}$. The rate of $0.9 \mathrm{~h}$ corresponds to a decreasing rate prior to attaining the pseudo-equilibrium state [49] (see Supplementary Data 'Assess pharmacokinetic parameters - Figure 2').

\section{Optimal protocol design \\ Sampling design}

As illustrated by the results of Yu et al., the possibility of describing various kinetic stages depends on the blood sampling protocol. To obtain accurate kinetic parameters, concentrations must be monitored until the total elimination of the substance. The sampling interval $\left(\mathrm{t}_{0} ; \mathrm{t}_{\mathrm{f}}\right.$; Table 1$)$ must permit the observation of the entire kinetic process and must not be ended too rapidly (Figure 5).

In clinical or research practice, we cannot take samples until substance has been entirely eliminated (until $t_{\infty}$ ). When deemed appropriate, blood collection stops at a determined time $\left(\mathrm{t}_{\mathrm{f}}\right)$. In the best configuration, $\mathrm{t}_{\mathrm{f}}$ corresponds to the time period when the concentration remains negligible, in other words, until the low limit of quantification (LLOQ). To obtain an exhaustive observation of the kinetics, sampling interval must be optimized during kinetic trials. In either case, it is necessary to obtain at least three concentrations to calculate the terminal slope. The importance and problems inherent in protocol blood sampling were underlined by Gibaldi et al. [50].

$\mathrm{Yu}$ et al. compared the first (rapid) phase with the HL obtained by Lo et al. [24] without hypothesizing or discussing the possibility that the second phase could be more representative of the elimination process. It would seem worthwhile to take at least the two HLs into account, especially if the HL could represent a biomarker for pregnancy-associated complications; in which case the second (slow phase) might be more informative. But they pointed out the fact that the discrepancies between the two results can be explained by the increased sensitivity of their detection method (leading to a bigger window sample). It is worthwhile considering the possible consequences of a longer monitoring period for pregnant women included in other studies [24,36].

Lau et al. [36] quantified a median HL of $114 \mathrm{~min}$ on seven patients (range: 46-210 min) and a median of $28 \mathrm{~min}$ on nine control women (range: 7-114 min) with log-linear regression. Theoretically, eight sample points should 
be available per patient for calculating the slope whereas for some of them, only three samples were available (see supplementary data of [36]). This is because the samples considered to compute HL are variable among the patients. The first sample point used for the log-linear regression could be 5-45 min postdelivery and the 'cut off' ( $\mathrm{t}_{\mathrm{f}}$ ), defined as the 'first nonzero trough concentration', could be 45, 60, 120 or 360 min depending on the patients.

These two limitations (limited number of sample points and restricted sampling interval) do not permit the possibility of describing a two-phase shape concentration-time curve (if any). Fatouros et al. [41] raised the problem of sample design in a physical exercise context. They concluded that sampling time after aseptic inflammation can lead to different conclusions regarding cfDNA responses.

\section{Quality of data}

Quality of data is essential to estimate accurate parameters. The more the concentrations represent 'true concentrations', the more the parameters will be right. To this end, the bioanalytical method must be validated for the appropriate matrix (human and/or animal plasma, blood, serum, etc.). The LLOQ should be as low as possible and the method must be sensitive and precise enough to allow the quantification of low concentrations. The latter are characteristic of the slow phase (elimination). For PK drug analysis, concentrations below the LLOQ are censored (considered as unreliable); in other words, the sample is not used for calculating parameters. In this review, we have only studied the evolution of measured cfDNA concentrations and not the bioanalytical methods used and their validation. However, we noted that $\mathrm{Yu}$ et al. [25] and Beiter et al. [37] were the only ones to specify a lower detection or quantification limit and did not use data providing important information [51]. Apart from under-LLOQ samples, all sample points should be used to calculate parameters and any sample point removed from the analysis needs to be justified. If we understand that there is a need to exclude some results (outliers), then this should be justified. Outliers may be considered as particular cases (rapid clearance, double peak, etc.) until further information is provided.

\section{What is the definition of a 'half-life'?}

'Half-life' is a familiar term very often used to describe the elimination of an entity, drugs or endogenous substances. A way that is often used to define or interpret biological HL is to consider HL as the time required to halve the plasma concentration. But this definition may be misleading and inappropriate because it assumes that the distribution pseudo-equilibrium is instantaneous [52]. In the PK field, the biological HL is the time required to halve the plasma concentration after reaching pseudo-equilibrium, meaning after homogenous distribution of the entity in the organism. This corresponds to the terminal log-linear curve. It is only in this context that HL can be a parameter representative of the elimination process (with one or more mechanisms included in the process). If we consider that a traumatic procedure (delivery, surgery, exercise, etc.) contributes to a sharp increase of cfDNA, we can expect plasma concentration have to reach a pseudo-equilibrium of distribution (biphasic curve). Thus, an incomplete monitoring of plasma concentration versus time or a less sensitive assay method leads to an underestimation of the terminal HL. On the contrary, different circumstances (e.g., lack of specificity of the bioanalysis method) could lead to an overestimation of HL.

\section{Conclusion \& future perspective}

Plasmatic cfDNA opens new perspectives in medical applications (prenatal diagnosis, SLE, cancer, etc.), knowing that its fluctuation rate provides interesting information for a biomarker. The results obtained to characterize the rate of elimination using calculated parameters are rare. The few reported data are discordant and no clear conclusion can be drawn. The heterogeneity of the cohorts used to determine cfDNA elimination characteristics can potentially explain the lack of concordance between the published data.

However, questions remain concerning the way to analyze the results in each context, and the definition given to the calculated parameters. Our review seems to highlight a lack of commune methodology to evaluate HL of elimination. As highlighted by a previous review concerning common biomarkers, the inconsistency of HL predictive values in various studies could be explained by the inconsistency of the methodologies used to calculate these HLs, leading to an inefficient use of reported kinetic parameters [47]. The same problem also occurs with cfDNA as a biomarker. Based on PK methodology, a kinetic analysis needs enough sample concentrations and an optimal design (accurate time sampling, suitable sampling interval) to match the parameters that are expected to be calculated. Moreover, even if $\mathrm{HL}$ is a standard parameter used in a biological context, we have seen that different slopes could be obtained the from $\mathrm{C}=\mathrm{f}(\mathrm{t})$ curve. If all slopes are correct, the interpretation of the calculated 
parameter could be totally different. The exclusive use of $\mathrm{HL}$ as a reflection of cfDNA evolution over time could set aside the influence of other physiological processes that could be involved in the fluctuation rate. Other authors have worked on all the processes simultaneously (distribution and elimination) by directly exploring the area under the concentration-time curve $\left[A U C_{0}^{\infty}\right]$ for cfDNA [53,54]. This could represent another interesting way to explore kinetics, on condition that the kinetic study is rigorously designed. In any case, absence of consensus concerning adequate methodologies used to assess kinetic biomarker parameters results in a collection of parameters with weak values, and in the future, a limited use in clinical practice.

\section{Executive summary}

Physiological \& pathological processes responsible for the cfDNA rate

- Cell-free DNA (cfDNA) plasmatic rate depends on two phenomena: distribution within the organism and elimination process.

- cfDNA has been shown to be an intercellular messenger and this suggests that it can travel from one site to another within the organism.

- The elimination pathway has not been clearly identified. But plasma nucleases and renal excretion seem to play a role.

- Different factors could influence the elimination of cfDNA: structure of cfDNA, DNA hydrolyzing activity and the binding to plasma proteins.

Kinetic parameters

- Results concerning kinetics of 'cfDNA' are rare and the main parameter explored in literature is half-life.

- Half-life was obtained in different physiological or pathological circumstances: treadmill exercise, hemodialysis, pregnancy and cancer.

Interpret the parameters

- Physiopathological state can influence the value of half-life but the methodology used to obtain half-life value could also influence this result.

- The definition considered for half-life, protocol design and quality of data have big consequences on the half-life values obtained for circulating cfDNA used as biomarker.

Conclusion \& future perspective

- Even if circulating cfDNA is considered to be a promising biomarker, it has not been translated into current clinical practice. This could be the result of insufficient information published concerning cfDNA kinetics.

- The exclusive use of half-life as a reflection of cfDNA evolution over time could set aside the influence of other physiological processes that could impact the fluctuation rate.

- Other possibilities to explore kinetics exist, for example, determination of the area under the concentration-time curve.

\section{Supplementary data}

To view the supplementary data that accompany this paper please visit the journal website at: www.future-science.com/doi/suppl /10.4155/fsoa-2017-0140.

\section{Author contributions}

S Khier contributed in the acquisition, analysis and interpretation of data, figures and table, and also contributed in critical revision as well as in the drafting of the manuscript. L Lohan contributed in the acquisition, analysis and interpretation of data, table, and also helped in drafting of the manuscript.

\section{Acknowledgements}

The authors would like to acknowledge J Brès (Professor of Pharmacokinetics) and S El Messaoudi for their advice and N Hargreaves (translator interpreter) for their proofreading.

Financial \& competing interests disclosure

The authors have no relevant affiliations or financial involvement with any organization or entity with a financial interest in or financial conflict with the subject matter or materials discussed in the manuscript. This includes employment, consultancies, honoraria, stock ownership or options, expert testimony, grants or patents received or pending, or royalties.

No writing assistance was utilized in the production of this manuscript. 
Open access

This work is licensed under the Creative Commons Attribution 4.0 License. To view a copy of this license, visit http://creativecomm ons.org/licenses/by/4.0/

\section{References}

1. Thierry AR, El Messaoudi S, Gahan PB, Anker P, Stroun M. Origins, structures and functions of circulating DNA in oncology. Cancer Metastasis Rev. 35(3), 347-376 (2016).

2. Fleischhacker M, Schmidt B. Circulating nucleic acids (CNAs) and cancer - a survey. Biochim. Biophys. Acta 1775(1), 181-232 (2007).

3. Lo YM, Corbetta N, Chamberlain PF et al. Presence of fetal DNA in maternal plasma and serum. Lancet Lond. Engl. 350(9076), 485-487 (1997).

4. Bettegowda C, Sausen M, Leary RJ et al. Detection of circulating tumor DNA in early- and late-stage human malignancies. Sci. Transl. Med. 6(224), $224 \mathrm{ra24}$ (2014).

5. Gold B, Cankovic M, Furtado LV, Meier F, Gocke CD. Do circulating tumor cells, exosomes and circulating tumor nucleic acids have clinical utility? A report of the association for molecular pathology. J. Mol. Diagn. JMD 17(3), 209-224 (2015).

6. Stroun M. Modifications des caractères à la suite de greffes intervariétales chez le Solanum melongena. CR Acad. Sci. Paris 255, 361-363 (1962).

7. Gahan PB, Chayen J, Silcox AA. Cytoplasmic localization of deoxyribonucleic acid in Allium cepa. Nature 195, 1115-1116 (1962).

8. Gahan PB, Chayen J. Cytoplasmic deoxyribonucleic acid. Int. Rev. Cytol. 18, 223-247 (1965).

9. Stroun M, Anker P. Nucleic acids spontaneously released by living frog auricles. Biochem. J. 128(3), P100-P101 (1972).

10. Stroun M, Anker P. Prehistory of the notion of circulating nucleic acids in plasma/serum (CNAPS): birth of a hypothesis. Ann. $N Y$ Acad. Sci. 1075, 10-20 (2006).

11. Gahan PB, Stroun M. The virtosome-a novel cytosolic informative entity and intercellular messenger. Cell Biochem. Funct. 28(7), 529-538 (2010).

12. Gahan PB, Perry IJ, Stroun M, Anker P. Effect of exogenous DNA on acid deoxyribonuclease activity in intact roots of Vicia faba L. Ann. Bot. 38(3), 701-704 (1974).

13. Pisetsky DS. The origin and properties of extracellular DNA: from PAMP to DAMP. Clin. Immunol. Orlando Fla. 144(1), 32-40 (2012).

14. Chelobanov BP, Laktionov PP, Vlasov VV. Proteins involved in binding and cellular uptake of nucleic acids. Biochemistry (Mosc.) 71(6), 583-596 (2006).

15. Vlassov VV, Laktionov PP, Rykova EY. Extracellular nucleic acids. BioEssays 29(7), 654-667 (2007).

16. Thakur BK, Zhang H, Becker A et al. Double-stranded DNA in exosomes: a novel biomarker in cancer detection. Cell Res. 24(6), 766-769 (2014).

17. Laktionov PP, Tamkovich SN, Rykova EY et al. Cell-surface-bound nucleic acids: free and cell-surface-bound nucleic acids in blood of healthy donors and breast cancer patients. Ann. NY Acad. Sci. 1022, 221-227 (2004).

18. Tamkovich SN, Bryzgunova OE, Rykova EY, Permyakova VI, Vlassov VV, Laktionov PP. Circulating nucleic acids in blood of healthy male and female donors. Clin. Chem. 51(7), 1317-1319 (2005).

19. György B, Szabó TG, Pásztói M et al. Membrane vesicles, current state-of-the-art: emerging role of extracellular vesicles. Cell. Mol. Life Sci. CMLS 68(16), 2667-2688 (2011).

20. Rykova EY, Morozkin ES, Ponomaryova AA et al. Cell-free and cell-bound circulating nucleic acid complexes: mechanisms of generation, concentration and content. Expert Opin. Biol. Ther. 12(Suppl. 1), S141-S153 (2012).

21. Gosse C, Le Pecq JB, Defrance P, Paoletti C. Initial degradation of deoxyribonucleic acid after injection in mammals. Cancer Res. 25(6), 877-883 (1965).

22. Chused TM, Steinberg AD, Talal N. The clearance and localization of nucleic acids by New Zealand and normal mice. Clin. Exp. Immunol. 12(4), 465-476 (1972).

23. Emlen W, Mannik M. Kinetics and mechanisms for removal of circulating single-stranded DNA in mice. J. Exp. Med. 147(3), 684-699 (1978).

24. Lo YM, Zhang J, Leung TN, Lau TK, Chang AM, Hjelm NM. Rapid clearance of fetal DNA from maternal plasma. Am. J. Hum. Genet. 64(1), 218-224 (1999).

25. Yu SCY, Lee SWY, Jiang P et al. High-resolution profiling of fetal DNA clearance from maternal plasma by massively parallel sequencing. Clin. Chem. 59(8), 1228-1237 (2013).

26. de Almeida EFP, Abdalla TE, Arrym TP et al. Plasma and urine DNA levels are related to microscopic hematuria in patients with bladder urothelial carcinoma. Clin. Biochem. 49(16-17), 1274-1277 (2016).

27. Salvi S, Martignano F, Molinari C et al. The potential use of urine cell free DNA as a marker for cancer. Expert Rev. Mol. Diagn. 16(12), 1283-1290 (2016). 
28. Yao W, Mei C, Nan X, Hui L. Evaluation and comparison of in vitro degradation kinetics of DNA in serum, urine and saliva: a qualitative study. Gene 590(1), 142-148 (2016).

29. Fenton K, Fismen S, Hedberg A et al. Anti-dsDNA antibodies promote initiation, and acquired loss of renal Dnase1 promotes progression of lupus nephritis in autoimmune (NZBxNZW)F1 mice. PLoS ONE 4(12), e8474 (2009).

30. Seredkina N, Zykova SN, Rekvig OP. Progression of murine lupus nephritis is linked to acquired renal Dnase1 deficiency and not to upregulated apoptosis. Am. J. Pathol. 175(1), 97-106 (2009).

31. Dewez B, Lans M, Allaeys V, Karaoglou A, Taper H, Roberfroid M. Serum alkaline deoxyribonuclease activity, a sensitive marker for the therapeutic monitoring of cancer patients: methodological aspects. Eur. J. Clin. Chem. Clin. Biochem. 31(11), 793-797 (1993).

32. Kragh-Hansen U, Chuang VTG, Otagiri M. Practical aspects of the ligand-binding and enzymatic properties of human serum albumin. Biol. Pharm. Bull. 25(6), 695-704 (2002).

33. Skvortsova TE, Bryzgunova OE, Lebedeva AO, Mak VV, Vlassov VV, Laktionov PP. Methylated cell-free DNA in vitro and in vivo. In: Circulating Nucleic Acids in Plasma and Serum. Gahan PB (Ed.). Springer, Dordrecht, The Netherlands, 185-194 (2010).

34. Zhang W, Wu J, Qiao B, Xu W, Xiong S. Amelioration of lupus nephritis by serum amyloid P component gene therapy with distinct mechanisms varied from different stage of the disease. PLoS ONE 6(7), e22659 (2011).

35. Rumore P, Muralidhar B, Lin M, Lai C, Steinman CR. Haemodialysis as a model for studying endogenous plasma DNA: oligonucleosome-like structure and clearance. Clin. Exp. Immunol. 90(1), 56-62 (1992).

36. Lau T-W, Leung TN, Chan LYS et al. Fetal DNA clearance from maternal plasma is impaired in pre-eclampsia. Clin. Chem. 48(12), 2141-2146 (2002).

37. Beiter T, Fragasso A, Hudemann J, Nieß AM, Simon P. Short-term treadmill running as a model for studying cell-free DNA kinetics in vivo. Clin. Chem. 57(4), 633-636 (2011).

38. Breitbach S, Sterzing B, Magallanes C, Tug S, Simon P. Direct measurement of cell-free DNA from serially collected capillary plasma during incremental exercise. J. Appl. Physiol. 117(2), 119-130 (2014).

39. Diehl F, Schmidt K, Choti MA et al. Circulating mutant DNA to assess tumor dynamics. Nat. Med. 14(9), 985-990 (2008).

40. Atamaniuk J, Vidotto C, Kinzlbauer M, Bachl N, Tiran B, Tschan H. Cell-free plasma DNA and purine nucleotide degradation markers following weightlifting exercise. Eur. J. Appl. Physiol. 110(4), 695-701 (2010).

41. Fatouros IG, Jamurtas AZ, Nikolaidis MG et al. Time of sampling is crucial for measurement of cell-free plasma DNA following acute aseptic inflammation induced by exercise. Clin. Biochem. 43(16-17), 1368-1370 (2010).

42. Fatouros IG, Destouni A, Margonis K et al. Cell-free plasma DNA as a novel marker of aseptic inflammation severity related to exercise overtraining. Clin. Chem. 52(9), 1820-1824 (2006).

43. Pertl B, Bianchi DW. Fetal DNA in maternal plasma: emerging clinical applications. Obstet. Gynecol. 98(3), 483-490 (2001).

44. Ette EI, Williams PJ. Population pharmacokinetics I: background, concepts and models. Ann. Pharmacother. 38(10), 1702-1706 (2004).

45. Mould DR, Upton RN. Basic concepts in population modeling, simulation and model-based drug development-part 2: introduction to pharmacokinetic modeling methods. CPT Pharmacomet. Syst. Pharmacol. 2, e38 (2013).

46. Mould DR, Upton RN. Basic concepts in population modeling, simulation and model-based drug development. CPT Pharmacomet. Syst. Pharmacol. 1, e6 (2012).

47. Almufti R, Wilbaux M, Oza A et al. A critical review of the analytical approaches for circulating tumor biomarker kinetics during treatment. Ann. Oncol. 25(1), 41-56 (2014).

48. Gliddon T, Salman S, Robinson JO, Manning L. Modeling C-reactive protein kinetic profiles for use as a clinical prediction tool in patients with Staphylococcus aureus bacteremia. Biomark. Med. 9(10), 947-955 (2015).

49. Jambhekar Sunil S, Breen Philip J. Basic Pharmacokinetics (2nd Edition). Pharmaceutical Press, London, UK (2012).

50. Gibaldi M, Weintraub H. Some considerations as to the determination and significance of biologic half-life. J. Pharm. Sci. 60(4), 624-626 (1971).

51. Senn S, Holford N, Hockey H. The ghosts of departed quantities: approaches to dealing with observations below the limit of quantitation. Stat. Med. 31(30), 4280-4295 (2012).

52. Toutain PL, Bousquet-Mélou A. Plasma terminal half-life. J. Vet. Pharmacol. Ther. 27(6), 427-439 (2004).

53. Holdenrieder S, Stieber P. Therapy control in oncology by circulating nucleosomes. Ann. NY Acad. Sci. 1022, 211-216 (2004).

54. Holdenrieder S, Stieber P, von Pawel J et al. Circulating nucleosomes predict the response to chemotherapy in patients with advanced non-small-cell lung cancer. Clin. Cancer Res. 10(18 Pt 1), 5981-5987 (2004). 
